\section{Os efeitos da intensificação no movimento da(s) mão(s) na produção de sinais da Libras}

The effects of intensification on hand(s)'s movement in the production of Libras signs

Thiago Steven dos SANTOS (UFPR) thiago.henryford@gmail.com André Nogueira XAVIER (UFPR) andrexavier@ufpr.br

Recebido em: 31 de jan. de 2019. Aceito em: 12 de jun. de 2019.
SANTOS, Thiago Steven dos; XAVIER, André Nogueira. Os efeitos da intensificação no movimento da(s) mão(s) na produção de sinais da Libras. Entrepalavras, Fortaleza, v. 9, n. 3, p. 57-75, set-dez/2019.

Resumo: Estudos a respeito do processo de intensificação nas línguas sinalizadas reportam o uso de expressões não-manuais e de mudanças no movimento em sua expressão (WILBUR; MALAIA; SHAY, 2012; e FUKS, 2016). Ao estudarem esse processo na Libras, levando em conta as fases do sinal (KITA; van GIJN; van der HULST, 1998), Santos e Xavier (2019) observaram, em algumas produções, alongamento do movimento não apenas na fase expressiva, mas também na fase de preparação para esta. Por meio da análise de sete dos 32 sinais coletados isoladamente por Xavier (2014) em suas formas basal e intensificada, produzidas três vezes por cada um dos 12 participantes surdos (seis homens e seis mulheres) da cidade de São Paulo, o presente trabalho tem como objetivo verificar, através de dados de duração, se a intensificação pode alongar o movimento não apenas da fase expressiva, mas também de outras fases da produção do sinal, a saber, a preparação e a retração (retorno ao repouso). A análise de cada uma dessas produções, assim como em Xavier (2017) e Santos e Xavier (2017), se deu através do software Elan, o qual permitiu a determinação do início e do fim de cada fase na produção dos sinais. Os resultados revelaram que, pelo menos para os dados analisados no presente trabalho, a intensificação afeta apenas a duração da fase expressiva.

Palavras-chave: Libras. Intensidade. Fases do sinal. 
v. 9 (3)

57-75

set-dez

2019

Abstract: Studies on the process of intensification in signed languages report the use of non-manual expressions and changes in movement in their expression (WILBUR, MALAIA, SHAY, 2012; FUKS, 2016). Santos and Xavier (2019) studied this process in Libras. By taking into account sign phases (KITA; van GIJN; van der HULST, 1998), they observed in some productions movement lengthening not only in the expressive phase, but also in the preparation phase. Through the analysis of seven of the 32 signs collected in isolation by Xavier (2014) in their baseline and intensified forms, produced three times by each of the 12 deaf participants (six men and six women) from the city of São Paulo, the present study aims to verify, through duration data, if intensification can affect not only the expressive phase, but also other phases of sign production, namely preparation and retraction (return to rest position). The analysis of each of these productions, as in Xavier (2017) and Santos and Xavier (2017), was done through the Elan software, which allowed for the determination of the beginning and the end of each phase in the production of the signs. The results revealed that, at least for the data analyzed in the present study, intensification affects only the duration of the expressive phase.

Keywords: Libras. Intensity. Sign phases.

\section{Introdução}

Benzinger (1971), ao analisar a expressão de intensidade na língua inglesa, demonstra que, além do uso de advérbios, existem outros recursos como alongamento da vogal e acento. Wilbur, Malaia e Shay (2012) investigaram o mesmo fenômeno na língua de sinais americana, ASL ${ }^{1}$. Segundo as autoras, a intensificação de adjetivos em ASL implica em realizar mudanças na sua forma, de maneira que eles fiquem visualmente distintos de sua forma basal. Na língua de sinais israelense, ISL ${ }^{2}$, esse processo foi analisado por Fuks (2016), de acordo com quem há diversas estratégias para expressar intensidade nessa língua, bem como a possibilidade de elas serem empregadas na intensificação de um mesmo sinal.

O primeiro estudo sobre intensidade em libras foi realizado por Xavier (2014) e teve como foco a duplicação de mãos como um dos recursos para a sua expressão. Tal estudo revelou que a duplicação não é obrigatória, mas apontou que mudanças nas características manuais e não-manuais dos sinais parecem ser. Xavier (2017) e Santos e Xavier (2017) avançaram na descrição do processo de intensificação na Libras, analisando essas mudanças e levando em conta as fases do sinal (KENDON, 1980; KITA; van GIJN; van der HULST, 1998).

Kendon (1980) analisa a estrutura da gesticulação, gestos manuais que co-ocorrem com a fala, e propõe que ela se organiza em

\footnotetext{
${ }^{1}$ Do inglês, American Sign Language.

2 Do inglês, Israeli Sign Language.
} 
três fases principais: preparação, golpe e retração. Kita, van Gijn e van der Hulst (1998), com base no trabalho seminal de Kendon, estendem essas fases à análise de línguas sinalizadas, diferindo deste, basicamente, na sua terminologia. Os referidos autores denominam como fase expressiva a fase nomeada como golpe por Kendon. A questão das fases do gesto/ sinal é retomada no estudo de Jantunen (2015). Ao investigar a duração dos sinais na língua de sinais finlandesa, ele propõe que, diferentemente da visão tradicional nos estudos sobre as línguas sinalizadas, os limites de um sinal não se restringem à sua fase expressiva. O autor demonstra que muitas informações articulatórias estão presentes bem antes do que geralmente é considerado o início dos sinais, ou seja, antes da fase expressiva, e continuam disponíveis mesmo depois que esta se encerrou, ou seja, durante a retração.

Em trabalho mais recente acerca da expressão de intensidade em libras, Santos e Xavier (2019) reportam, além das diversas modificações manuais e não-manuais nas produções da forma intensificada, alteração no movimento correspondente à fase de preparação do sinal ALÍVIO. A partir disso, este trabalho objetiva examinar se a expressão de intensidade pode afetar, além da fase expressiva, outras fases do sinal, especificamente, o seu movimento.

Para isso, o presente artigo foi organizado da seguinte maneira: nas duas próximas seções serão apresentadas as pesquisas que analisaram o fenômeno de intensidade nas línguas de sinais e os estudos a respeito das fases dos gestos e sinais. Na seção metodologia, serão descritos os procedimentos utilizados na análise dos dados investigados. Finalmente, serão apresentados os resultados e, na sequência, nossas considerações finais.

\section{Intensidade nas línguas sinalizadas}

Em línguas orais, tais como português e inglês, a expressão de intensidade, de modo geral, é realizada através de intensificadores como 'muito' e 'very', respectivamente (XAVIER, 2017, p.1). Porém, como demonstrado por Benzinger (1971), na língua inglesa, além do uso de advérbios, existem outros recursos como alongamento de vogal e o acento. Nas línguas de sinais, esse fenômeno também vem sendo explorado por alguns pesquisadores. Wilbur, Malaia e Shay (2012) investigaram a expressão de intensidade na ASL e, em seu estudo, ao 
v. 9 (3)

57-75

set-dez

2019

compararem a forma basal ${ }^{3}$ de alguns adjetivos dessa língua com a sua forma intensificada, identificaram mudanças, como aumento na tensão da face e das mãos, acréscimo ou aumento na trajetória do movimento, soltura retardada ${ }^{4}$, franzimento da face e inclinação da cabeça.

Mais recentemente, Fuks (2016) investigou o mesmo processo na ISL. A autora examinou dados de intensidade produzidos em contexto por nove sinalizantes surdos, filhos de pais surdos e, que, portanto, têm a ISL como primeira língua. No referido estudo, os surdos sinalizaram a partir de um texto escrito em hebraico, no qual apareciam os adjetivos 'ocupado', 'chato', 'magro', 'lotado', 'mimado', 'bravo', 'esperto', 'frio', 'difícil', 'calmo' e 'triste', acompanhados pelos advérbios 'muito' e 'tão'.

Na análise de 56 casos de intensificação, Fuks observou que os sinalizantes utilizaram três principais advérbios intensificadores da ISL: MUITO, REALMENTE/VERDADE e COMPLETAMENTE. MUITO foi usado em 70\%, REALMENTE/VERDADE, em 25\% e COMPLETAMENTE, em $5 \%$ dos casos de intensificação.

Além da intensificação sintática, a autora observou 11 modificações na forma dos adjetivos quando intensificados: (1) acréscimo, (2) alongamento, (3) omissão, (4) substituição e (5) repetição do movimento, (6) alongamento da suspensão, mudança (7) na localização, (8) na orientação, (9) na localização com movimentos repetidos, (10) tensionamento e (11) substituição da configuração de mão.

Em relação à Libras, o primeiro estudo sobre intensidade, realizado por Xavier (2014), focou na duplicação de mãos. O autor analisou 32 sinais produzidos por 12 sujeitos surdos (seis do sexo masculino e seis do sexo feminino), nascidos e residentes na cidade São Paulo. De acordo com os resultados, 50\% dos sujeitos que participaram do estudo duplicaram o número de mãos pelo menos uma vez quando produziram a forma intensificada de pelo menos um dos 32 sinais analisados. Os outros 50\% nunca o fizeram. Sendo assim, Xavier concluiu que a duplicação de mãos para expressar intensidade não é obrigatória. Apesar disso, o autor observou que todos os sujeitos, ao realizarem a forma intensificada dos sinais, produziram mudanças em alguns parâmetros articulatórios (manuais e não-manuais).

\footnotetext{
3 Prefere-se aqui o termo 'forma basal' à designação 'forma não intensificada' ou neutra, para abranger casos em que a intensificação incide sobre formas intensificadas.

4 Suspensão da mão por mais tempo do que o habitual antes do início do movimento do sinal.
} 
Mais recentemente, Xavier (2017) avançou na descrição do processo de intensificação em Libras por meio da reanálise de dados de dois de 12 sujeitos que participaram do experimento reportado no estudo de 2014. Mais precisamente, os dados analisados pelo autor foram constituídos por três produções de 32 sinais, em sua forma isolada, em duas condições: basal e intensificada. A análise de tais produções foi realizada no programa Elan5, através do qual foram anotadas modificações nos parâmetros articulatórios manuais (configuração de mão, orientação da palma, localização, movimento e o número de mãos) e não-manuais (sobrancelhas, olhos, bochechas, boca, cabeça e tronco).

Os resultados apresentados por Xavier mostraram uma grande variedade de recursos utilizados para expressar intensidade em libras. $\mathrm{O}$ autor identificou, na forma intensificada dos sinais analisados, o uso de sobrancelhas franzidas, bochechas infladas, tronco inclinado, mudanças na localização, na configuração de mão, na localização, no movimento, na orientação, no número de mãos e, na duração do sinal.

Subsequentemente, Santos e Xavier (2017) analisaram três produções dos sinais de CHUVA e EXPERIÊNCIA também nas condições basal e intensificada, realizadas por 12 sujeitos. Os autores selecionaram alguns dos aspectos manuais e não-manuais analisados por Xavier (2017). Dentre os não-manuais, examinaram os comportamentos da sobrancelha, olhos e bochechas. Já entre os manuais, analisaram a localização, a repetição e o aumento da trajetória do movimento.

A análise da forma intensificada dos sinais CHUVA e EXPERIÊNCIA revelou o emprego de sobrancelhas franzidas, de olhos semicerrados, de bochechas infladas, de expansão da localização e de aumento da trajetória em sua produção. Com relação à duração dos sinais analisados, os autores observaram que a forma intensificada durou mais do que a forma basal. Essa diferença resultou estatisticamente significativa. Porém, ao testar especificamente a repetição do movimento, uma das possíveis explicações para o aumento da duração dos sinais intensificados, Santos e Xavier concluíram que, apesar de, em alguns casos, haver aumento no número de repetições do movimento na forma intensificada, esse recurso não resultou estatisticamente significativo.

O estudo de Santos e Xavier (2019) teve como objetivo dar prosseguimento à descrição da expressão de intensidade em libras

${ }^{5}$ Software livre que permite anotar e transcrever, manual e semi-automaticamente gravações de áudio ou vídeo. (Site para download: https://tla.mpi.nl/tools/tla-tools/ elan/download/). 
v. 9 (3)

57-75

set-dez

2019

iniciadas por Xavier (2014, 2017) e Santos e Xavier (2017). Para tanto, foi realizada uma reanálise dos sinais CHUVA e EXPERIÊNCIA e, a análise dos sinais NÃO-SABER, FÁCIL, SOFRER, VONTADE e ALÍVIO, produzidos pelos 12 sujeitos que participaram do estudo de Xavier (2014).

Os autores observaram, como em trabalhos anteriores, que a intensidade pode ser expressa por diversos aspectos não-manuais e manuais. O uso das sobrancelhas franzidas e do alongamento do movimento foram os mais utilizados pelos sujeitos. Apesar disso, mesmo esses recursos não foram utilizados na realização da forma intensificada de todos os sinais analisados, o que sugere a sua não obrigatoriedade. Vale destacar que nesse estudo o aumento da duração na forma intensificada também resultou estatisticamente significativo. Santos e Xavier observaram interação entre a estratégia de intensificação e a presença de contato no sinal ALÍVIO. Provavelmente por ser realizado com a mão contactando permanentemente a testa (bem como por seu movimento consistir em mudança de configuração de mão, logo não envolver deslocamento espacial desta), os autores observaram que o alongamento do movimento se deu, em alguns casos, no movimento que leva a mão para a testa, ou seja, antes do que tradicionalmente se consideraria o início do sinal (JANTUNEN, 2015). Especificamente, observaram alteração no movimento correspondente à fase de preparação do sinal e não apenas na sua fase expressiva (KITA; van GIJN; van der HULST, 1998).

A partir dessa observação, o presente trabalho tem como objetivo verificar se, assim como nos casos reportados por Santos e Xavier (2019), a intensificação pode afetar não apenas a fase expressiva (tradicionalmente referida como correspondente ao sinal), mas também outras fases da produção do sinal, a saber, a preparação e a retração (retorno da(s) mão(s) ao repouso). Especificamente, objetivase verificar, através de dados de duração, se o movimento das referidas fases é alongado, assim como já observado na fase expressiva. Para tanto, serão analisados sete sinais (CHUVA, EXPERIÊNCIA, NÃO-SABER, FÁCIL, SOFRER, VONTADE e ALÍVIO) produzidos pelos 12 sujeitos que participaram do estudo de Xavier (2014).

\section{0 gesto/sinal e suas fases}

Nas interações face-a-face, as pessoas, concomitantemente à fala, também realizam gestos com o corpo. Os gestos realizados pelos braços e pelas mãos são designados por Kendon (1980) como gesticulação. 
Segundo o autor, em uma gesticulação, as mãos normalmente são levantadas e posicionadas à frente do corpo, onde realizam alguns movimentos e retornam para a posição de repouso. Ao analisar a estrutura da gesticulação, o autor propõe que ela se organiza em três fases principais. A primeira, denominada preparação, consiste na ação da(s) mão(s) que a(s) prepara para o gesto propriamente dito, ou seja, o golpe. Essa segunda fase também pode ser seguida de uma suspensão, em que a mão permanece na forma e na posição alcançada no fim do golpe. A essa fase segue a retração, na qual a mão começa a retornar para a posição de repouso. Kendon menciona que pode haver também uma retração parcial, caso no qual a mão não retorna totalmente para a posição de repouso (KENDON, 1980, p. 212-213).

Com base no trabalho seminal de Kendon, outros pesquisadores avançaram na análise das fases dos gestos (DUNCAN, s/d; MCNEILL, 2005; SEYFEDINIPUR, 2006). Alguns deles ampliaram, inclusive, seu escopo ao considerá-las na análise de línguas sinalizadas (KITA; van GIJN; van der HULST, 1998). Como indica a tabela 1, baseada em Bressem e Ladewig (2011), as fases originalmente propostas por Kendon perpassam quase todos os trabalhos subsequentes ao de Kendon.

Quadro 1 - Fases do gesto/sinal estabelecidas pelos autores

\begin{tabular}{|c|l|l|l|l|l|}
\hline & $\begin{array}{c}\text { Kendon } \\
(1980: \text { 212) }\end{array}$ & $\begin{array}{c}\text { Kita, van Gijn } \\
\text { e van der } \\
\text { Hulst (1998: } \\
\text { 26, 29) }\end{array}$ & $\begin{array}{c}\text { Duncan } \\
\text { (s.d) }\end{array}$ & $\begin{array}{c}\text { McNeill } \\
\text { (2005: 31) }\end{array}$ & $\begin{array}{c}\text { Seyfeddinipur } \\
\text { (2006) }\end{array}$ \\
\hline $\begin{array}{c}\text { Posição de repouso } \\
\text { (rest position) }\end{array}$ & & & & & \\
\hline $\begin{array}{c}\text { Preparação } \\
\text { (preparation) }\end{array}$ & & & & & \\
\hline $\begin{array}{c}\text { Preparação } \\
\text { interrompida } \\
\text { (interrupted } \\
\text { preparation/stroke) }\end{array}$ & & & & & \\
\hline $\begin{array}{c}\text { Suspensão pré- } \\
\text { golpe } \\
\text { (pre-stroke hold) }\end{array}$ & & & & & \\
\hline \begin{tabular}{c} 
Golpe (stroke) \\
\hline $\begin{array}{c}\text { Suspensão pós- } \\
\text { golpe } \\
\text { (post-stroke hold) }\end{array}$
\end{tabular} & & & & & \\
\hline $\begin{array}{c}\text { Golpe em suspensão } \\
\text { (stroke hold) }\end{array}$ & & & & & \\
\hline Suspensão (hold) & & & & & \\
\hline
\end{tabular}


v. 9 (3)

57-75

set-dez

2019

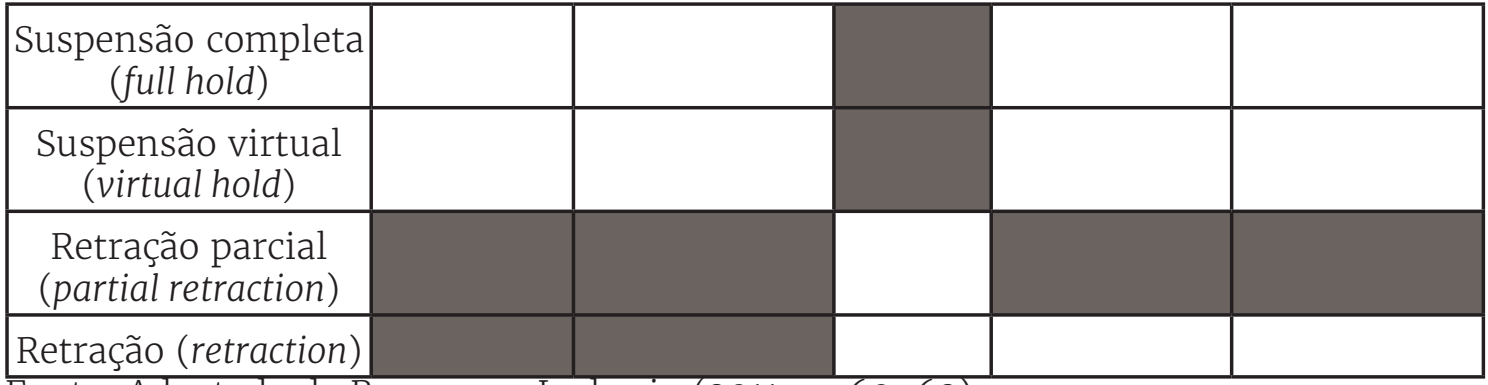

Fonte: Adaptada de Bressem e Ladewig (2011, p. 60-62)

Como é possível observar na tabela acima, todos os autores concordam com relação à presença de um golpe em um gesto. No entanto, há algumas divergências quanto à ocorrência e à natureza de algumas fases. Seyfeddinipur (2006), por exemplo, propõe a preparação interrompida. Duncan (s/d) comenta que existem golpes que são realizados com a(s) mão(s) imóvel(is), denominados por ele de golpe em suspensão. O autor propõe ainda a suspensão completa e a suspensão virtual, nas quais a mão fica, respectivamente, totalmente estática, ou com algum movimento, porém, mantendo a forma e a localização do espaço onde o gesto foi realizado. Kita, van Gijn e van der Hulst (1998) e McNeil (2005) propõem as fases de suspensão pré e pós-golpe. Segundo os autores, ambas têm a finalidade de retardar o ápice ou o fim do golpe e assim permitir seu alinhamento temporal com a parte da fala a que ele deve se referir (BRESSEM; LADEWIG, 2011, p. 60-62).

Os fonólogos das línguas de sinais têm tido como principal foco de investigação apenas o movimento que, nos termos dos estudos do gesto, corresponderia ao golpe, ou fase expressiva (KITA; van GIJN; van der HULST, 1998, p. 3). Segundo Jantunen (2015), isso deve também ser reflexo da perspectiva fonológica de Stokoe (1960) e seus seguidores, de acordo com a qual o sinal é definido como uma unidade estruturalmente simultânea. As bordas, ou seja, o movimento inicial (fase de preparação) e final (fase de retração) não são, portanto, consideradas partes do sinal.

A mudança de uma perspectiva estritamente fonológica para uma perspectiva fonética no que concerne aos limites de um sinal se inicia com o trabalho de Kita, van Gijn e van der Hulst (1998). Como já dito, os autores propõem uma organização sintagmática das fases do movimento tanto para a gesticulação quanto para as línguas de sinais. As fases propostas, em essência, são as mesmas já definidas por Kendon (1980) e McNeil (2005) neste artigo.

Na mesma linha, Jantunen (2015) discute a questão de onde o sinal começa e termina. Ao analisar a literatura sobre a fonologia das 
línguas de sinais, o autor conclui que, mesmo em modelos como o de Movimento e Suspensão de Liddell (1984), em que, contrariamente a Stokoe, defende-se que os sinais têm uma estrutura sequencial, logo, são tomados como unidades temporalmente organizadas, as bordas também não são consideradas parte destes (p. 96-101)6.

No estudo de Jantunen acerca da duração de sinais na língua de sinais finlandesa, o autor relata que muitas informações articulatórias, especialmente a orientação e a configuração de mão, estão presentes antes do que geralmente é considerado o início dos sinais. Precisamente, antes do começo da chamada fase expressiva, a qual, por sua vez, geralmente tem como marco inicial a chegada da mão à localização em que o sinal é ou começa a ser produzido e a consequente mudança na direção de seu movimento. O autor aponta que tais componentes articulatórios também permanecem acessíveis depois da produção da fase expressiva, ou seja, durante a fase de retração. Com base nisso, ele afirma que os sinais devem ser tratados como unidades mais longas do que geralmente são. Como ilustração, ele apresenta uma produção isolada, quadro a quadro, do sinal ENVIAR da língua de sinais finlandesa (Figura 1).

${ }_{6}$ Para um estudo acerca da estrutura interna de sinais da Libras à luz da versão subsequente do modelo de Liddell (1984), ver Xavier (2006). 
v. $9(3)$

57-75 set-dez 2019

66

Figura 1 - Produção isolada frame a frame do sinal ENVIAR da língua de sinais finlandesa.
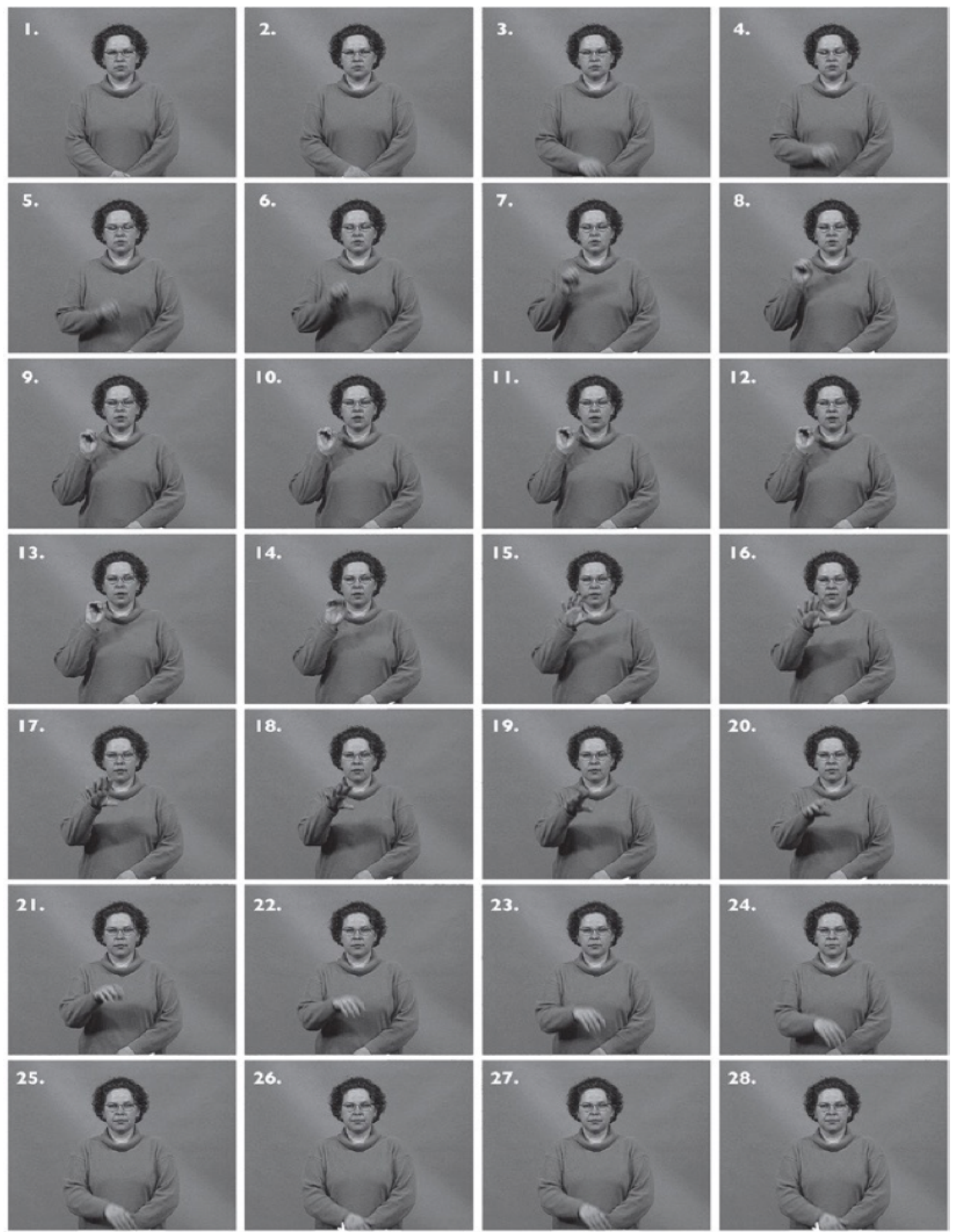

Fonte: Reproduzida de Jantunen (2015, p. 107)

De acordo com Jantunem (2015), o domínio fonético do sinal, tendo como base os autores que limitam o sinal à fase expressiva, corresponderia ao intervalo entre os frames 10-17. No entanto, como se pode observar, características articulatórias que compõem o sinal não 
estão presentes pela primeira vez apenas no frame 10. Além disso, o frame 17 não é o último em que essas características são observadas. Conforme explica, a configuração de mão inicial do sinal está completamente formada no frame 5 e a configuração de mão final permanece ativa até o frame 20. De maneira análoga, a orientação de mão produzida no sinal já se apresenta no frame 7 e traços dela são detectáveis até o frame 19. Sendo assim, o autor conclui que, se tal sinal fosse segmentado levando em consideração o aparecimento e o desaparecimento de suas características articulatórias, ele duraria aproximadamente duas vezes.

Conjugando os estudos sobre as fases do gesto na produção de sinais de línguas sinalizadas com o trabalho de Jantunen (2015) sobre a extensão destes, este estudo tem como objetivo averiguar se, assim como nos casos reportados por Santos e Xavier (2019), a intensificação pode afetar não apenas o movimento da fase expressiva, mas também de outras fases da produção de sinais da Libras, a saber, a preparação e a retração.

\section{Metodologia}

As análises realizadas neste trabalho se basearam em sete dos 32 sinais coletados por Xavier (2014), a saber, CHUVA (Figura 2)7, EXPERIÊNCIA (Figura 3), NÃO-SABER (Figura 4), ALÍVIO (Figura 5), VONTADE (Figura 6), FÁCIL (Figura 7) e SOFRER (Figura 8), tanto em sua forma basal, quanto na intensificada, produzidas por 12 sujeitos $(1 \times 2 \times 7 \times 12=168)$. A seleção dos sinais levou em consideração a presença ou não de contato na forma de citação e, no primeiro caso, o tipo de contato: inicial, permanente ou final. Assim, foram selecionados dois sinais sem contato com o corpo (CHUVA e EXPERIÊNCIA), dois sinais com contato inicial (NÃO-SABER e FÁCIL), dois sinais com contato permanente (ALÍVIO e VONTADE) e um sinal com contato final (SOFRER). Objetivou-se com essa seleção observar a interação entre essa característica articulatória e as estratégias de intensificação.

7 Por razões de restrição de espaço, foram utilizados um ou dois frames medial(is) da fase expressiva da forma basal para ilustrar a produção dos sinais 
v. 9 (3)

57-75

set-dez

2019
Figura 2 - CHUVA

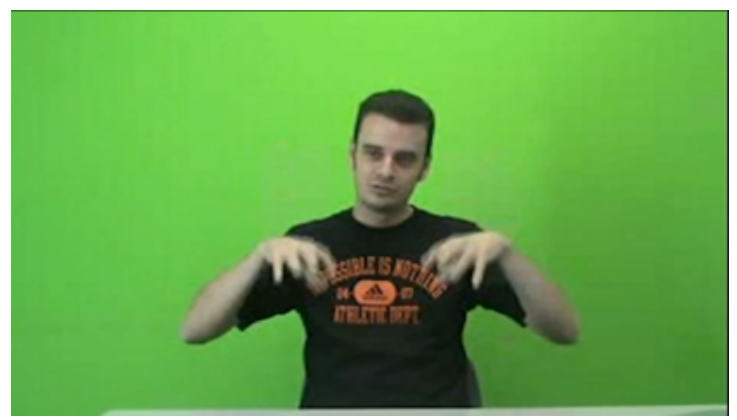

Fonte: Produzida pelos autores.

Figura 4 - NÃO-SABER

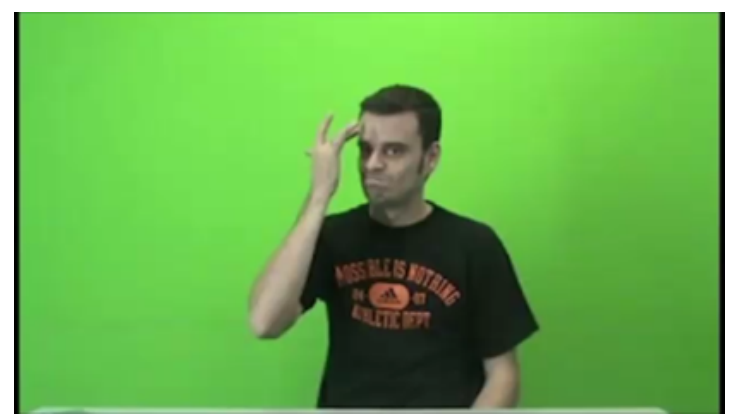

Fonte: Produzida pelos autores.

Figura 5 - ALÍVIO

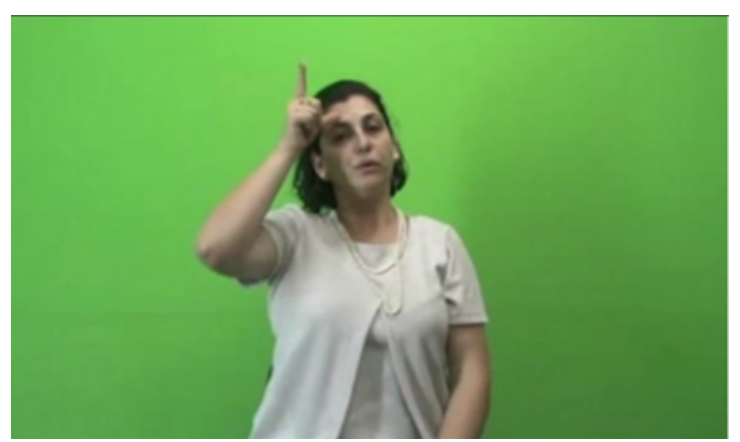

Fonte: Produzida pelos autores.

Figura 7 - FÁCIL
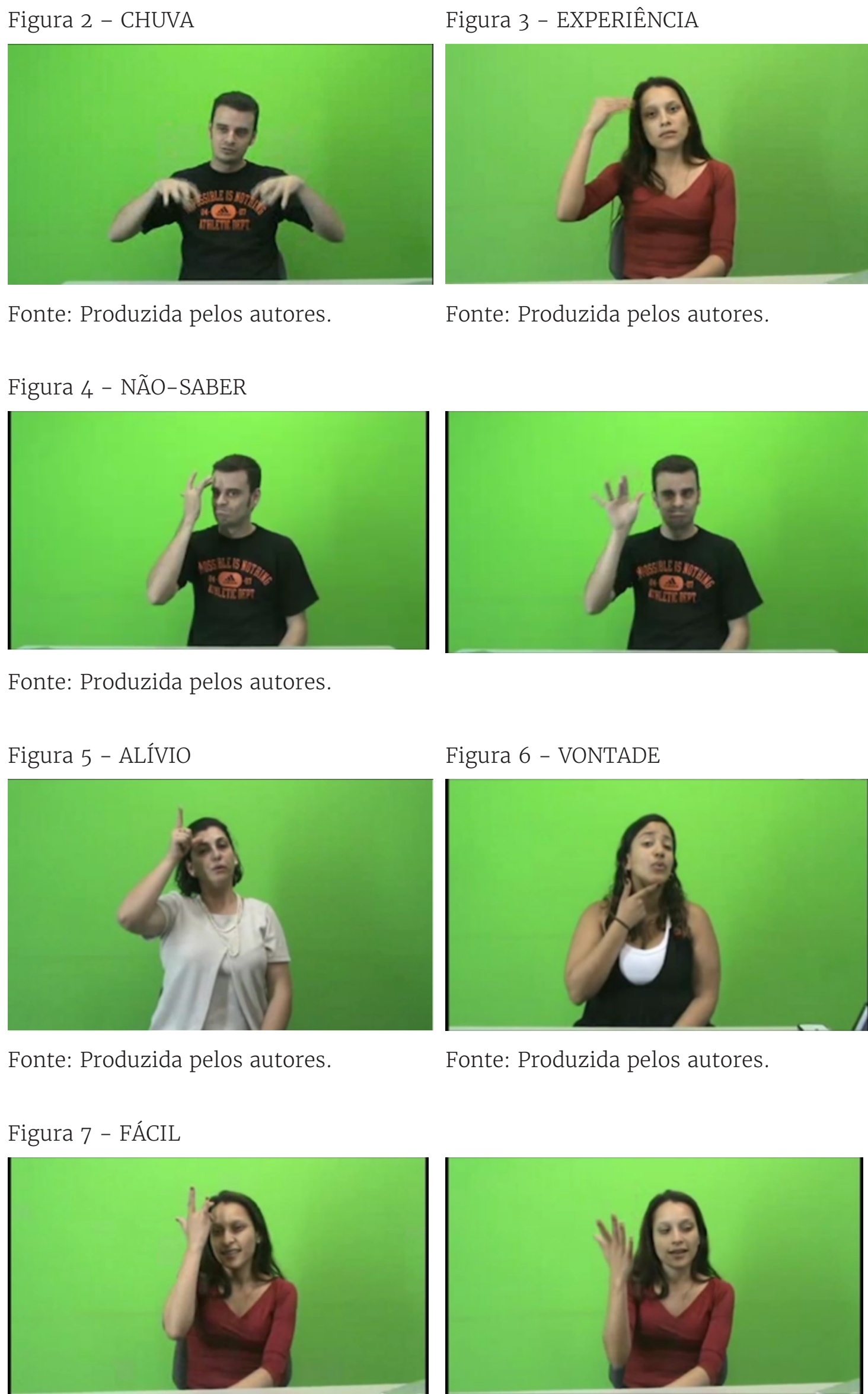

Fonte: Produzida pelos autores.

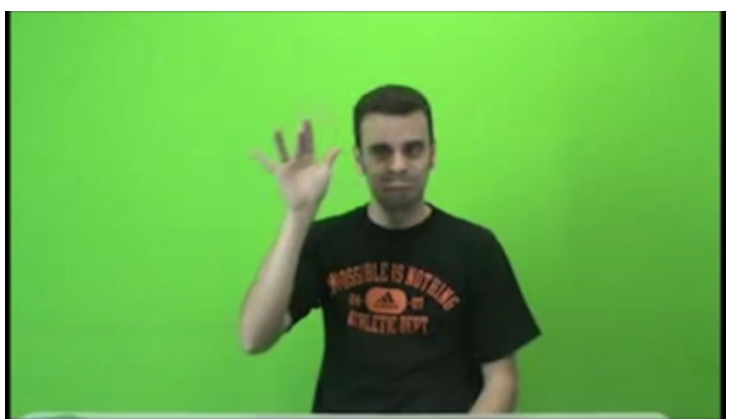

Figura 6 - VONTADE

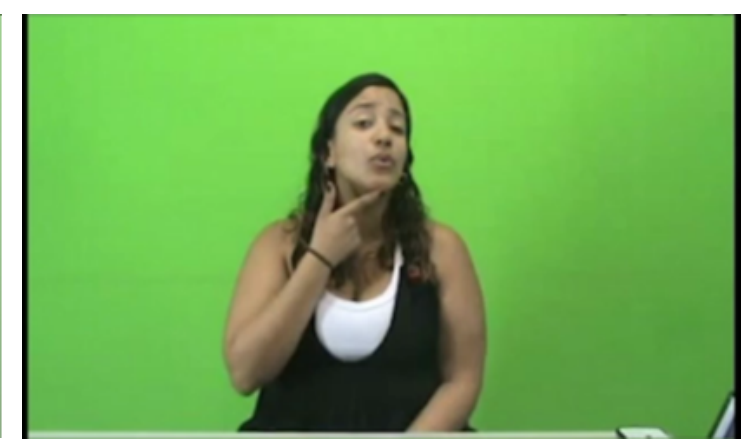

Fonte: Produzida pelos autores.

Fonte: Produzida pelos autores. 
Figura 8 - SOFRER

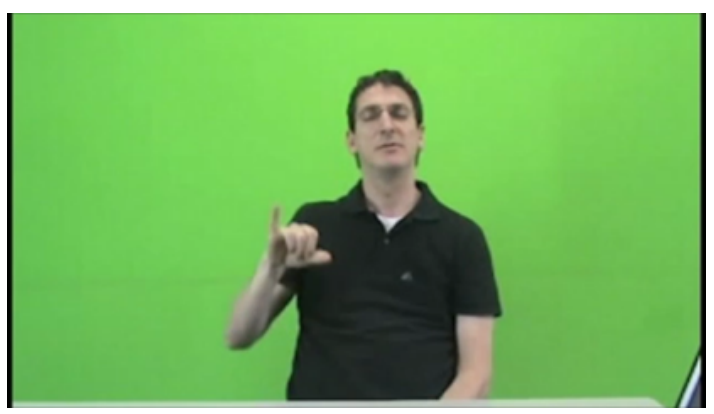

Fonte: Produzida pelos autores.

No corpus de Xavier (2014), as três produções de cada sinal estavam originalmente separadas por sujeito e em ordem aleatória. Por meio do programa MovieMaker, foi extraída a segunda dessas três produções, as quais foram reunidas, por sinal, em um único arquivo de vídeo. Esse procedimento facilitou a análise através do software Elan, o qual permitiu a segmentação, isto é, a demarcação do início e do fim de cada produção. Essa segmentação seguiu os critérios discutidos por Xavier, Tkachman e Gick (2015), de acordo com os quais o início do sinal corresponde ao primeiro frame em que é possível perceber a mão ou o braço do sinalizante saindo do estado de repouso. Para a delimitação do fim da fase de preparação, início e fim da fase expressiva, bem como o fim da fase de retração, foi considerado o frame em que há uma mudança na direção do movimento.

Os dados foram analisados quantitativamente, por meio do teste estatístico Wilcoxon realizado no $\mathrm{R}^{8}$. Esse teste permitiu comparar a duração de cada uma das fases dos sinais na sua forma basal com a respectiva duração na forma intensificada.

\section{Resultados}

Os dados de alguns dos sujeitos analisados, referidos neste trabalho por meio das duas primeiras letras de seus nomes, tiveram de ser excluídos. Entre as razões está o fato de que a participante CI não contrastou, de forma significativa, a produção que supostamente seria intensificada da forma basal ${ }^{9}$. Outra razão envolveu a produção referente

\footnotetext{
${ }^{8}$ WILCOXON, F. Individual comparisons by ranking methods. Biometrics Bulletin, 1(6), 80-83. 1945. Disponível em: https://www.r-project.org/. Acesso em: 17 dez. 2019.

9 Diferentemente dos demais participantes do estudo de Xavier (2014), além da ausência de mudanças nas características do movimento do sinal e nas expressões faciais, a duração daquela que deveria ser a forma intensificada foi muito próxima da forma basal.
} 
v. 9 (3)

57-75

set-dez 2019

ao sinal EXPERIÊNCIA realizado por LU, que empregou um sinal que, apesar de sinônimo, difere daquele esperado por Xavier (2014). Justificase da mesma forma a exclusão das produções realizadas por CR1, CR2, e DA para o sinal FÁCIL. As produções das formas basal e intensificada de LU dos sinais de CHUVA, SOFRER e VONTADE foram sinalizadas sem pausa entre elas, o que dificultou o estabelecimento de seus limites. Uma vez que os dados aqui analisados se baseiam em produções isoladas e não em sinalização contínua, essas produções foram desconsideradas para a mensuração da duração das fases. Dessa forma, das 168 produções inicialmente consideradas, foram efetivamente analisados 146 dados.

A tabela 2 abaixo mostra as médias e os desvios-padrão das durações da fase de preparação, levando em consideração as produções das formas basal e intensificada de cada um dos sinais. Embora a diferença seja mínima, observa-se que a média da forma intensificada de todos os sinais, com exceção do sinal FÁCIL, é maior do que a da forma basal correspondente. Os valores obtidos para o desvio-padrão, em sua maioria, também indicam uma diferença entre a fase de preparação da forma basal e a da forma intensificada.

Tabela 2 - Média e desvio-padrão da duração em ms da fase de preparação por sinal

\begin{tabular}{|c|c|c|c|c|}
\hline & \multicolumn{2}{|c|}{ BASAL } & \multicolumn{2}{c|}{ INTENSIFICADA } \\
\cline { 2 - 5 } & Média & Desvio-padrão & Média & Desvio-padrão \\
\hline ALÍVIO & 0.69 & 0.14 & 0.82 & 0.23 \\
\hline CHUVA & 0.45 & 0.04 & 0.51 & 0.08 \\
\hline EXPERIÊNCIA & 0.44 & 0.09 & 0.45 & 0.08 \\
\hline FÁCIL & 0.54 & 0.03 & 0.53 & 0.06 \\
\hline NÃO-SABER & 0.45 & 0.05 & 0.50 & 0.17 \\
\hline SOFRER & 0.30 & 0.07 & 0.35 & 0.10 \\
\hline VONTADE & 0.39 & 0.08 & 0.41 & 0.08 \\
\hline
\end{tabular}

Fonte: Produzida pelos autores.

Semelhantemente à fase de preparação, a duração da fase expressiva na forma intensificada, em média, foi maior do que a da forma basal (Tabela 3). Essa diferença é corroborada pelos valores do desvio-padrão maiores do que os da forma basal correspondente, em quatro dos sete sinais. 
Tabela 3 - Média e desvio-padrão da duração em ms da fase expressiva por sinal

\begin{tabular}{|c|c|c|c|c|}
\hline & \multicolumn{2}{|c|}{ BASAL } & \multicolumn{2}{c|}{ INTENSIFICADA } \\
\cline { 2 - 5 } & Média & Desvio-padrão & Média & Desvio-padrão \\
\hline ALÍVIO & 0.97 & 0.48 & 1.37 & 0.70 \\
\hline CHUVA & 1.16 & 0.35 & 1.70 & 0.42 \\
\hline EXPERIÊNCIA & 1.18 & 0.30 & 1.50 & 0.44 \\
\hline FÁCIL & 0.72 & 0.23 & 0.95 & 0.70 \\
\hline NÃO-SABER & 0.42 & 0.29 & 0.56 & 0.29 \\
\hline SOFRER & 1.43 & 0.54 & 1.79 & 0.39 \\
\hline VONTADE & 1.09 & 0.42 & 1.46 & 0.36 \\
\hline
\end{tabular}

Fonte: Produzida pelos autores.

A tabela 4, por sua vez, mostra as médias e os desvios-padrão das durações da fase de retração. A média da forma intensificada foi maior em quatro dos sete sinais analisados. No entanto, assim como na fase de preparação, a diferença entre essas médias não é tão pronunciada. Já em relação ao desvio-padrão, não se observa um padrão nos resultados obtidos, dado que há um caso em que as formas basal e intensificada apresentaram valores idênticos, três em que a forma intensificada apresentou valores menores e três, em que esta apresentou valores maiores que a basal.

Tabela 4 - Média e desvio-padrão da duração em ms da fase de retração por sinal

\begin{tabular}{|c|c|c|c|c|}
\hline & \multicolumn{2}{|c|}{ BASAL } & \multicolumn{2}{c|}{ INTENSIFICADA } \\
\cline { 2 - 5 } & Média & Desvio-padrão & Média & Desvio-padrão \\
\hline ALÍVIO & 0.55 & 0.14 & 0.55 & 0.14 \\
\hline CHUVA & 0.47 & 0.15 & 0.44 & 0.11 \\
\hline EXPERIÊNCIA & 0.48 & 0.08 & 0.50 & 0.07 \\
\hline FÁCIL & 0.67 & 0.33 & 0.72 & 0.15 \\
\hline NÃO-SABER & 0.52 & 0.23 & 0.71 & 0.33 \\
\hline SOFRER & 0.48 & 0.10 & 0.54 & 0.13 \\
\hline VONTADE & 0.44 & 0.12 & 0.47 & 0.26 \\
\hline
\end{tabular}

Fonte: Produzida pelos autores.

No que diz respeito ao impacto da intensificação sobre as fases do sinal, foi realizado o teste Wilcoxon $(\alpha=0,5)$ para verificar se as diferenças observadas nos valores sumarizados nas tabelas 2-4 são estatisticamente significativas. Como se pode ver na tabela 5 abaixo, a 
v. 9 (3)

57-75

set-dez 2019

fase de preparação resultou significativa apenas para as produções do sinal CHUVA. Com relação à fase expressiva, o teste resultou significativo para o sinal CHUVA e VONTADE. Já para a fase de retração, o resultado indica que não há diferença entre a forma basal e a forma intensificada nessa fase.

Tabela 5 - Valor de p obtido através do teste Wilcoxon ( $\alpha: 0,05)$ por sinal

\begin{tabular}{|c|c|c|c|}
\hline Sinal/Fase & Preparação & Expressiva & Retração \\
\hline ALÍVIO & 0.09395 & 0.8955 & 0.1513 \\
\hline CHUVA & 0.04491 & 0.005196 & 0.65 \\
\hline EXPERIÊNCIA & 0.8497 & 0.1903 & 0.3533 \\
\hline FÁCIL & 0.8744 & 0.6454 & 0.2345 \\
\hline NÃO-SABER & 0.693 & 0.3086 & 0.09323 \\
\hline SOFRER & 0.3066 & 0.08921 & 0.2408 \\
\hline VONTADE & 0.9705 & 0.04326 & 0.7959 \\
\hline Fonte: Produzida pelo autor. & &
\end{tabular}

A tabela 3 mostra que na fase expressiva há uma diferença entre a duração da forma basal e intensificada mais relevante do que nas outras fases. No entanto, a tabela 5 mostra que, para essa fase, o resultado foi significativo apenas para dois dos sete sinais analisados. Tal fato pode-se explicar pela pequena quantidade de dados. Para checar se, com um maior número de dados, as diferenças seriam estatisticamente significativas, realizou-se o mesmo teste, comparando-se, agora, as durações das formas basal e intensificada dos sete sinais aqui analisados. O mesmo foi feito com as durações obtidas para as demais fases. Com isso, como se pode observar na tabela 6 a seguir, apenas a diferença na duração da fase expressiva resultou estatisticamente significativa, fato que é corroborado pelos boxplots na figura 9.

Tabela 6 - Valores obtidos através do teste Wilcoxon ( $($ ::0,05) por fase

\begin{tabular}{|c|c|c|c|}
\hline & Fase de preparação & Fase expressiva & Fase de retração \\
\hline Valor de p & 0.2897 & 0.0008059 & 0.1419 \\
\hline
\end{tabular}

Fonte: Produzida pelos autores. 
Figura 9 - Boxplots para duração da fase de preparação (a), fase expressiva (b) e fase de retração (c) de todos os sinais

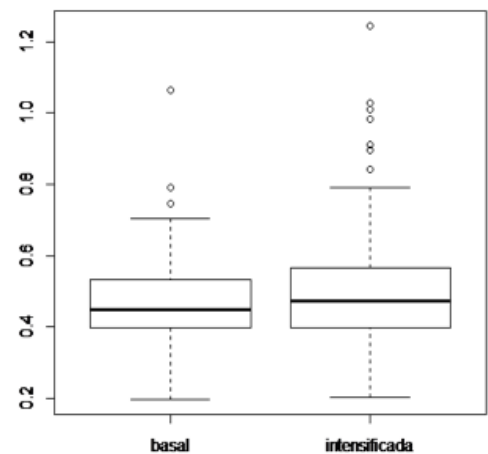

(a)

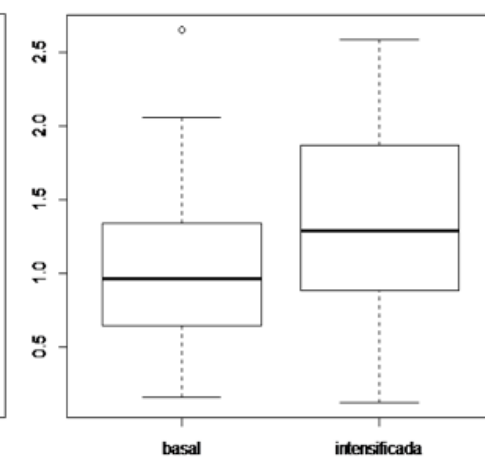

(b)

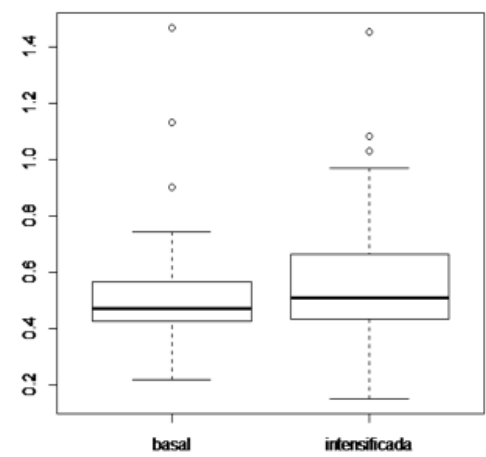

(c)

Fonte: Produzida pelos autores.

\section{Considerações Finais}

Este estudo teve como objetivo averiguar se, assim como nos casos reportados por Santos e Xavier (2019), a intensificação pode afetar não apenas o movimento da fase expressiva, mas também de outras fases da produção de sinais da Libras, a saber, a preparação e a retração. Conforme os resultados obtidos, é possível concluir que, para os dados analisados no presente trabalho, a intensidade aparentemente afeta apenas o movimento da fase expressiva. Com relação à fase de preparação e retração, os resultados mostraram que as produções da forma intensificada duraram mais do que a forma basal, no entanto, essa diferença não foi confirmada estatisticamente.

Jantunen (2015), em seu estudo a respeito da duração de sinais na língua de sinais finlandesa, faz uma crítica a Stokoe (1960) e a Liddell (1984) por não considerarem em seus trabalhos as bordas como parte do sinal. O autor aponta que muitas informações articulatórias, especialmente a orientação e a configuração de mão, estão presentes antes do que geralmente é considerado o início dos sinais, isto é, na fase de preparação, bem como depois do que tradicionalmente é tomado como seu fim, ou seja, a sua retração.

Com base nos resultados de nossa análise da intensificação em Libras, aparentemente, as bordas não parecem ser relevantes à discussão sobre a delimitação do sinal para além da fase expressiva, já que ela não parece incidir significativamente sobre elas. Por outro lado, com base em Santos e Xavier (2019), estratégias de intensificação podem ser observadas na preparação, fato que corrobora a argumentação de Jantunen em relação à incorporação das bordas aos limites dos sinais. 
v. 9 (3)

57-75

set-dez

2019

\section{Referências}

BENZINGER, E. Intensifiers in Current English. 1971. Tese (Doutorado) University of Florida, 1971.

BRESSEM, J.; LADEWIG, H. S. Rethinking gesture phases: Articulatory features of gestural movement?. Semiotica 184-1/4, p. 53-91. 2011.

DUNCAN, S. D. Coding "manual”. Disponível: <http://mcneilllab.uchicago. edu/pdfs/Coding_Manual.pdf>. Acesso em: 28 de nov. de 2018.

FUKS, O. Intensifier actions in Israeli Sign Language. Gesture. v.2, n.15, p. 192-223. 2016.

JANTUNEN, T. How long is the sign? Linguistics, 53:1, p. 93-124. 2015.

KENDON, A. Gesticulation and speech: Two Aspects of the Process of Utterance. In: KEY, M. R. The Relation Between Verbal and Nonverbal Communication. The Hague: Mouton, 1980. 207-227.

KITA, S.; van GIJN, I.; van der HULST, H. Movement phases in signs and cospeech gestures, and their transcription by human coders. Gesture and sign language in human-computer interaction, Springer Berlin Heidelberg, p. 2335. 1998.

LIDDELL, S. K. THINK and BELIEVE: Sequentially in American Sign Language signs. Language, 60. Vol 2. 372-399. 1984.

McNEILL, D. Gesture and thought. Chicago: Chicago University Press, 2005.

SANTOS, T. S.; XAVIER, A. N. . Modificação dos parâmetros articulatórios na expressão de intensidade em Libras. In: SEMANA DE LETRAS DA UFPR, 19., 2018, Curitiba. Cadernos da Semana de Letras. Curitiba: Setor de Humanas, 2017. v. 1. p. 29-40.

SANTOS, T. S.; XAVIER, A. N. Recursos manuais e não-manuais na expressão de intensidade em libras. Leitura, 63, p. 120-137, 2019.

STOKOE, W. Sign Language Structure: An Outline of the Visual Communication Systems of the American Deaf". Studies in Linguistics: Occasional Papers, 8, Washington: Gallaudet University Press, 1960.

SEYFEDDINIPUR, M. Disfluency: Interrupting speech and gesture (MPI Series in Psycholinguistics 29). Nimegen: University of Nijmegen, 2006.

WILBUR, R. B.; MALAIA, E.; SHAY, R. A. Degree modification and intensification in American sign language adjectives. Lecture Notes in Computer Science (Including Subseries Lecture Notes in Artificial Intelligence and Lecture Notes in Bioinformatics), 7218, p. 92-101. 2012

XAVIER, A. N. A expressão de intensidade em Libras. Intercâmbio, v. 36, p. $1-25,2017$. 
XAVIER, A. N. Uma ou duas? Eis a questão! Um estudo do parâmetro número de mãos na produção de sinais da língua brasileira de sinais (libras). 2014. (Tese Doutorado em Linguística). Instituto de Estudos da Linguagem, Universidade Estadual de Campinas, Campinas, 2014.

XAVIER, A. N. Descrição fonético-fonológica dos sinais da língua brasileira de sinais (libras). 2006. 175 p. Dissertação (Mestrado em Linguística). Departamento de Linguística, Universidade de São Paulo, São Paulo, 2006.

XAVIER, A. N.; TKACHMAN, O.; GICK, B. Towards convergence of methods for speech and sign segmentation. In: ACOUSTICS WEEK IN CANADA, 2015, Hallifax. Anais [...]. Hallifax: Acoustics Week in Canada, 2015. 